\title{
Dedicated X-Ray Mapping System with Single and Multiple SDD Detectors for Quantitative X-Ray Mapping and Data Processing
}

\author{
Richard Wuhrer ${ }^{1}$ and Ken Moran ${ }^{2}$ \\ ${ }^{1}$ University of Western Sydney, Advanced Materials Characterisation Facility (AMCF), Australia \\ ${ }^{2}$ Moran Scientific Pty Ltd, 4850 Oallen Ford Road, Bungonia, NSW, 2580, Australia
}

A JEOL 840 SEM/EDS system has been converted into a dedicated X-Ray Mapping (XRM) instrument, due to the needs of many users from varying disciplines including materials science and engineering, biology, geology and environmental science. The system performs 24 hours-7 days per week XRM electron microscopy (XRMEM). The system has just been upgraded with an Amptek 123 FAST123 silicon drift detector (SDD) with an Ultra Thin C1 window to allow for faster mapping. The XRMEM is setup to maximize its ability to operate with a larger working distance so that the instrument can perform x-ray maps at very low magnifications and high pixel resolution (1024x1024). This is now requested by a large number of users. With the use of the high count rate detectors, such as the Amptek SSD, our research team is collecting XRM's from over 3 hours and up to several days; this is especially true when trace elemental analysis and mapping is required.

Quantitative X-ray mapping (QXRM) using multi-detectors and combined detectors, such as WDS/EDS, not only reduces mapping time, but also improves the ability to map minor and trace elements very accurately. One interesting outcome of this is that we now need to improve the collection efficiency of our WDS detectors to enable them to perform at these lower beam currents [1-3]. To take advantage of this increased precision and sensitivity of QXRM, careful attention to all those parameters necessary for high quality analysis is required. Important characteristics such as resolution, gain zero calibration, pulse pileup and other artifacts need to be well characterized for each detector used. Images are then created from the fully background corrected, overlap corrected and inter-element corrected calculation at each pixel in the image. As there is a very large dynamic concentration range to be considered (5 orders of magnitude) it is important to understand that the precision at each level will depend on how well this characterization is carried out [1]. To achieve this, time is no longer an issue but a means to accomplish a more precise result. Thus, if we can introduce more efficient detection then we can achieve this result in a shorter time. This may be achieved by counting more x-rays with one detector, as long as the critical quantitative characteristics of the detector is not compromised, or this can easily be achieved by adding more detectors.

For QXRM to work correctly for multi-detector systems, the characteristics of each detector must be accurately determined so that the final quantification of the individual detectors can be summed. To accomplish this more effectively, the full spectrum at each pixel for each SDD detector should be saved. As a final check for consistency between detectors, a technique was developed called "Colouring Verification Technique (CVT)" that involves assigning a different RGB colour for each detector for the same element (Fig. 1). When combining the three maps of the same element, a grey scale map should be obtained (Fig. 1), not a colour map. This indicates a total correlation between the three detectors at the most critical final stage of quantification. Thus, the use of multiple detectors is a good way to determine which errors may be inherent in the specimen, the mathematical processing; and the detector systems. The use of this colouring technique for multiple detectors has also been found to be very valuable when analysing rough samples.

We have further developed this colouring technique to show differences between raw intensity maps and quantification maps (Quantification Performance Test-QPT) [3]. In Fig. 2, the chromium Region 
of Interest (ROI) has been made red, the stripped intensity map is green and the quantitative map is blue. We can clearly see colouring around the boundaries of the tungsten carbide particles with the matrix, indicating that the results from the three processes are different. This is a classic example of why we need to quantify the results, for the difference between the intensity profile and the quantification profile is caused by a large atomic number correction on the chromium by tungsten ( $\mathrm{Z}$ correction). We have confirmed this by plotting an intensity profile and the quantitative profile across this region (Fig. 2).
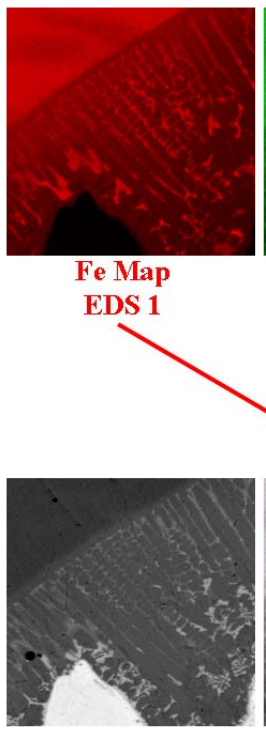

BSE Image

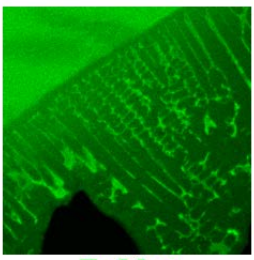

Fe Map EDS 2
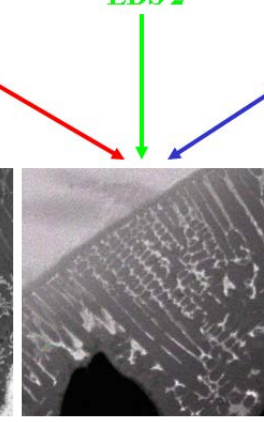

RGB Image

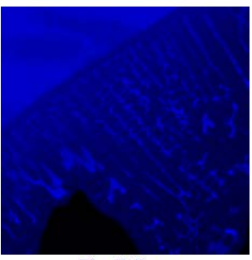

Fe Map EDS 3

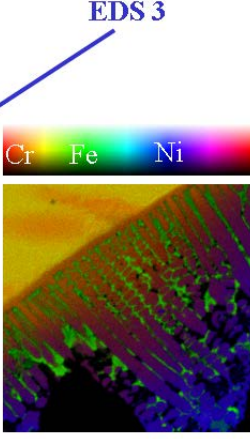

Pseudo Image

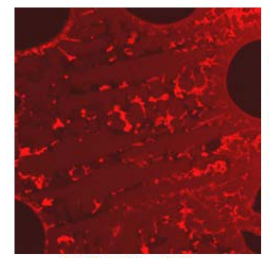

ROI Cr Map

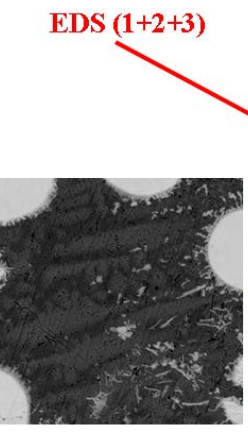

BSE Image

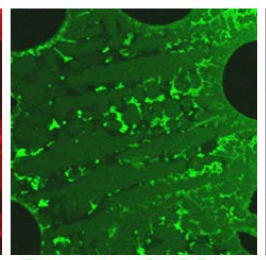

Intensity Cr Map Quantitative Cr Map

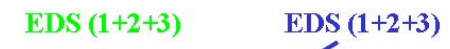

Fig. 1: Quantitative chromium X-ray maps taken with detector 1 (red), detector 2 (green), detector 3 (blue), RGB images detector 1, 2 and 3 combined and pseudo colour map from the summed quantitative images of the three detectors, showing nickel as blue [1]. $\mathrm{HWOF}=100 \mu \mathrm{m}$.

Fig. 2: A different RGB colour is assigned to the combined sum of all detectors for an individual element. The RGB image shows a grey scale map, slightly coloured, indicating miss- correlation between the three elements. Also shown is the pseudo image for the three elements present (Ni, $\mathrm{Cu}, \mathrm{Cr})$ [3-4]. $\mathrm{HWOF}=110 \mu \mathrm{m}$.

Our research aims at developing post processing techniques to improve the quantitation of X-ray map data and to develop further post processing techniques for improved characterisation as an aid in assessing the practical properties of complex materials [4]. This also includes developing techniques of handling X-ray mapping data collected from multiple X-ray detectors around the column.

\section{References}

[1] K. Moran and R. Wuhrer, "Quantitative Bulk and Trace Element X-Ray Mapping Using Multiple Detectors”, Mikrochimica Acta, Vol. 155, pp. 59-66 (2006).

[2] K. Moran and R. Wuhrer, "X-ray Mapping and Interpretation of Scatter Diagrams", Mikrochimica Vol. 155, pp. 209-217 (2006).

[3] R. Wuhrer, K. Moran and M. R. Phillips, "Multi-Detector X-Ray Mapping and Generation of Correction Factor Images for Problem Solving“, Microscopy and Microanalysis, 14(suppl 2), 1108CD-1109CD (2008).

[4] R. Wuhrer, K. Moran and M. R. Phillips,”X-Ray Mapping and Post Processing“, Microscopy and Microanalysis, 12(suppl 2), 1404CD-1405CD (2006). 